\title{
Structure of nitrous oxide-carbon dioxide alloys
}

\author{
A.A. Solodovnik and V.V. Danchuk \\ B. Verkin Institute for Low Temperature Physics and Engineering of the National Academy of Sciences of Ukraine \\ 47 Lenin Ave., Kharkov 61103, Ukraine \\ E-mail: solodovnik@ilt.kharkov.ua
}

Received December 12, 2008

\begin{abstract}
Structure of $\mathrm{N}_{2} \mathrm{O}-\mathrm{CO}_{2}$ solid mixtures was investigated by transmission electron diffraction over the entire range of their mutual concentrations. In the temperature range $5-65 \mathrm{~K}$ the experimental data indicate no phase separation in these alloys. The average structure of the solutions belongs to the Pa3 symmetry. The behavior of the concentration dependence of the lattice parameter has a nonmonotonic character, displaying a sharp step, the positions of which on the concentration scale depends on the deposition temperature. A possible explanation of this phenomenon is suggested.
\end{abstract}

PACS: $78.55 . \mathrm{Kz}$ Solid organic materials; 61.05.J-Electron diffraction and scattering.

Keywords: molecular cryocrystals, $\mathrm{N}_{2} \mathrm{O}-\mathrm{CO}_{2}$ alloys, electron diffraction.

\section{Introduction}

The interest towards solid solution of $\mathrm{CO}_{2}-\mathrm{N}_{2} \mathrm{O}$ is mainly associated with the investigation of the phase transitions due to the molecule disorientation and the formation of an orientational glass state [1]. A glass-like character of the phonon scattering on low-energy excitations was found in the crystalline $\mathrm{N}_{2} \mathrm{O}$ [2]. Solid $\mathrm{N}_{2} \mathrm{O}$ and $\mathrm{CO}_{2}$ belong to the class of molecular cryocrystals consisting of linear molecules and are physically very similar. The $\mathrm{N}_{2} \mathrm{O}$ $(\mathrm{N}-\mathrm{N}-\mathrm{O})$ molecule differs from $\mathrm{CO}_{2}(\mathrm{O}-\mathrm{C}-\mathrm{O})$ one by a symmetry and dipole moment. Both the crystals have the cubic (fcc) lattice in which the axes of the molecules are directed along one of the four spatial diagonals of the cube ( $\mathrm{Pa} 3$ space group) at the equilibrium vapor pressure. Such a structure is stable over the entire range of an existence of the solid phase up to the triple point. In the case of nitrous oxide the molecules are «head-tail» disoriented and because a long-range order is absent ( $\mathrm{Pa} 3$ symmetry are observed «on average»). A possible dipolar ordering may take place below $11 \mathrm{~K}$ according to the estimates [3]. In this case the structure of the phase must be $P 2_{l} 3$ and it means that an ordering of molecular ends occurred in the lattice. However in the structural studies [4,5] the disordered «head-tail» model agrees better with experimental data than the ordered one. A partial dipolar ordering reveals itself in peculiarities of the temperature dependence of the Grüneisen constant for solid $\mathrm{N}_{2} \mathrm{O}$ [6]. It can be ex- plained by the formation of clusters in which the $P 2{ }_{1} 3$ structure is locally realized. An absence of the end-to-end order may be due to a low rate of molecular reorientation. Hence, the dipole disorder occurring at high temperatures becomes kinetically frozen at lower temperatures. The same problem of «head-tail» order exists in solid $\alpha$-CO. Owing to the interaction between dipole moments the structure of the $\alpha$-CO crystal should belong to $P{ }_{1} 3$ space group at sufficiently low temperatures. It follows from calculations [3] whereas the structural data [5] show that the ends of molecules are randomly oriented. The mechanism of «head-tail» flips in solid $\mathrm{N}_{2} \mathrm{O}$ and $\alpha$-CO was studied [7] and shown that $\mathrm{N}_{2} \mathrm{O}$ crystal has no short-range dipolar order while solid $\alpha$-CO has. The $\mathrm{N}_{2} \mathrm{O}$ molecules might reorient with the aid of some intrinsic defect. Like the $\mathrm{N}_{2} \mathrm{O}$ and $\mathrm{CO}_{2}$ pair, solid carbon monoxide and nitrogen have the close physical parameters except for the symmetry of the molecules. To consider the intermolecular interactions producing differently local potential barriers, the orientational ordering was studied in the solid mixtures $\mathrm{N}_{2}-\mathrm{CO}$ by optical spectroscopy $[8,9]$. The difference in the dynamics of both molecules remains even in the «ideal» $\mathrm{N}_{2}-\mathrm{CO}$ mixtures. Much is known about the effects of dilution of spherically symmetric atoms in a molecular crystal matrix. It has been established that the rare-gas doping lowers the potential barrier hindering the rotation of molecules $[10,11]$. The binary systems in which the both molecular components are 
substances with a strong anisotropic interaction between particles, have not investigated. One can expect surprising effects for the mixtures of triatomic crystals. Moreover the choice of the $\mathrm{N}_{2} \mathrm{O}-\mathrm{CO}_{2}$ system is dictated by the following circumstance. There are no reliable data about the character of the phase diagram of these components. The $\mathrm{N}_{2} \mathrm{O}-\mathrm{CO}_{2}$ solid mixtures containing $25,50,75 \mathrm{~mol} . \% \mathrm{CO}_{2}$ were studied by the x-ray diffraction method [12]. The purpose was to establish regions of the solid solution and to determine their structure parameter.

\section{Experimental}

Investigations were carried out by transmission high-energy electron diffraction (THEED) using helium cryostat. The specimens were prepared directly in situ by quench depositing room-temperature gas mixtures of known composition simultaneously onto a composite substrate which consisted of two parallel strips made of different materials, amorphous carbon and fine grained polycrystalline aluminum. We kept the total amount of the mixture deposited in each experiment the same. Al substrate film also served as the internal standard. The presence of this standard makes it possible to carry out precise measurements of the lattice parameter $a$ when the photographic technique is used for registering the diffraction pattern. The relative error of measurement was usually about $0,1 \%$. For weak and highly blurred reflections, the error was twice as large. We used $99,99 \%$ pure gases in our investigations. The composition of the investigated mixtures was varied within the entire range of mutual concentrations from 0 to 1 . During the whole diffraction experiment both intensity and focusing of the electron beam did not change. Measurements were made in temperature range between 5 and $65 \mathrm{~K}$. Deposition took place at substrate temperatures of 5 and $65 \mathrm{~K}$. The high-temperature deposition was aimed at realizing a state closest to the equilibrium state in the samples, while the purpose of low-temperature deposition was to obtain amorphous films if it is possible. Annealing of such films could provide information about the structural phases adjoining the solidus line on the phase diagram. The experimental technique for electron diffraction measurements in the range of cryogenic temperatures has been described in detail elsewhere [13].

\section{Results}

We shall now describe the experimental results and analyze them.

For a substrate temperature of $65 \mathrm{~K}$ during condensation, the deposited specimens were in the form of polycrystalline samples with a large grain size. The deposits did not show any marked signs of a texture. Any preferable orientation was considered absent if rotation of the sample around a horizontal axis lying in its plane did not disturb of the intensity distribution along diffraction rings. The coherent scattering region on the substrate plane usually varied between 100 and $150 \AA$. Typical diffraction patterns for the investigated binary alloys are shown in Fig. 1 indicating no phase separation in the solid mixtures at $65 \mathrm{~K}$. For whole concentration range all diffraction peaks belong to the $\mathrm{Pa} 3$ space group. The character of obtained diffraction patterns did not change as the samples were cooled from 65 to $5 \mathrm{~K}$ and reheated to $65 \mathrm{~K}$ and did not varied with the composition of the mixtures being deposited. All the peaks of Pa3 symmetry were
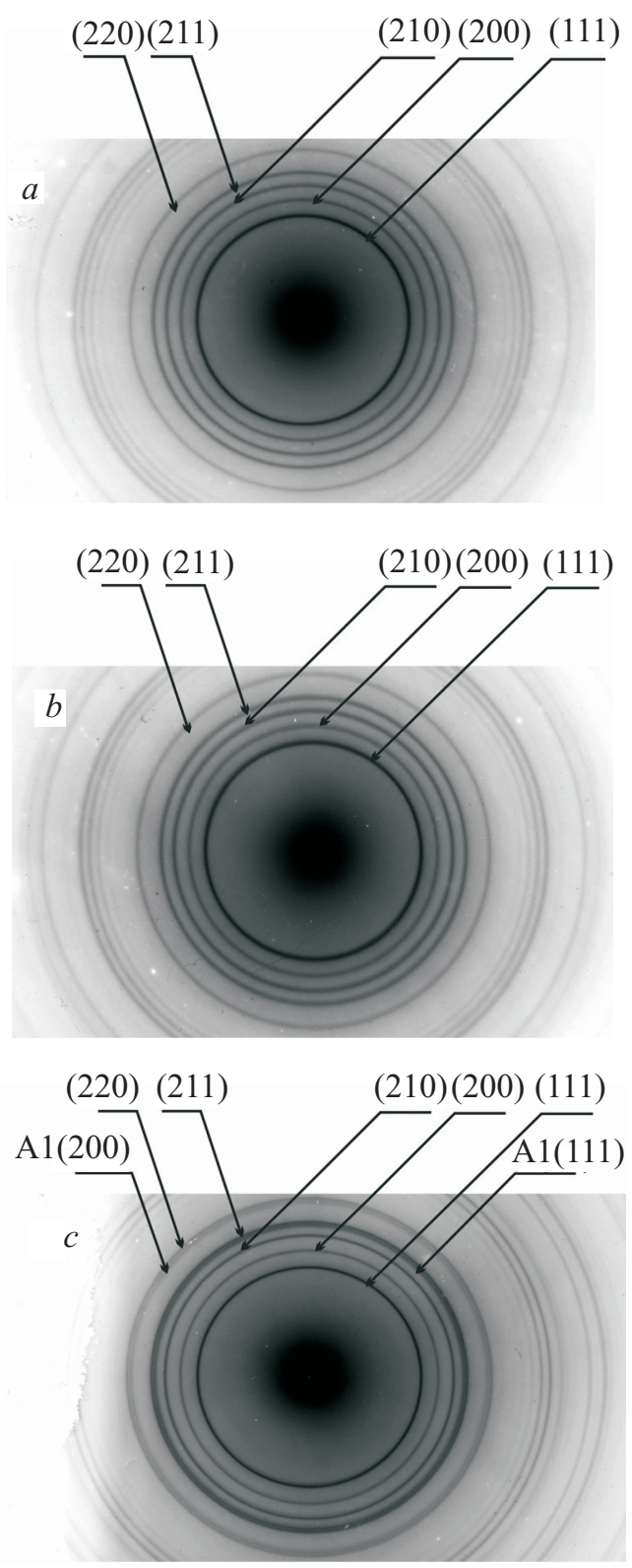

Fig. 1. Electron diffraction patterns from $\mathrm{CO}_{2}-\mathrm{N}_{2} \mathrm{O}$ alloys. Deposition temperature 65 K. 25 mol. $\% \mathrm{CO}_{2}$ on carbon substrate $(a)$. Equimolar mixture on carbon substrate $(b) .95 \mathrm{~mol}$. $\% \mathrm{CO}_{2}$ on $\mathrm{Al}$ substrate $(c)$. 
clearly manifested for the high temperature samples irrespective of conditions used.

The most correct information about the concentration range for an existence of solid solutions can be obtained from an analysis of the dependence of the lattice parameter $a$ on the composition of the mixtures. The concentration dependence is shown in Fig. 2. The lattice parameter was determined at $65 \mathrm{~K}$ after holding the freshly prepared condensates at this temperature for several minutes. It can be seen from Fig. 2 that as the carbon dioxide concentration increases the lattice parameter of the condensate decreases. Hence the behavior of the observed concentration dependence of $a$ gives evidence for a single-phase structure of alloys and mutual solubility. We used Vegard's dependence for obtaining a rough estimate of the possible variation of $a$ in the solutions. For the systems containing less than $10 \%$ impurities the experimental results are in rather good agreement with the theoretical ones. The values of Vegards's dependence differ and lie below the experimentally obtained values for $10-30 \%$ and $70-90 \% \mathrm{CO}_{2}$ range. It can mean that the substitution of matrix molecules for impurity is accompanied by a considerable loosening of the matrix lattice. One of the bright peculiarities of the concentration dependence (see Fig. 2) is that, the curve displays a step in the values of lattice parameter in the narrow concentration interval between 46 and 50 mol. \% $\mathrm{CO}_{2}$. This range divides $\mathrm{N}_{2} \mathrm{O}$-based and $\mathrm{CO}_{2}$-based solutions. If we suggest that a phase separation process takes a place, we would obtain two systems of diffraction rings. In this case the overlap of two patterns must lead to the broadening of the lines by $35 \%$ according our estimates. However the reflections had weak tendency to broadening and its experimental value did not exceed $15 \%$ within this content range. The

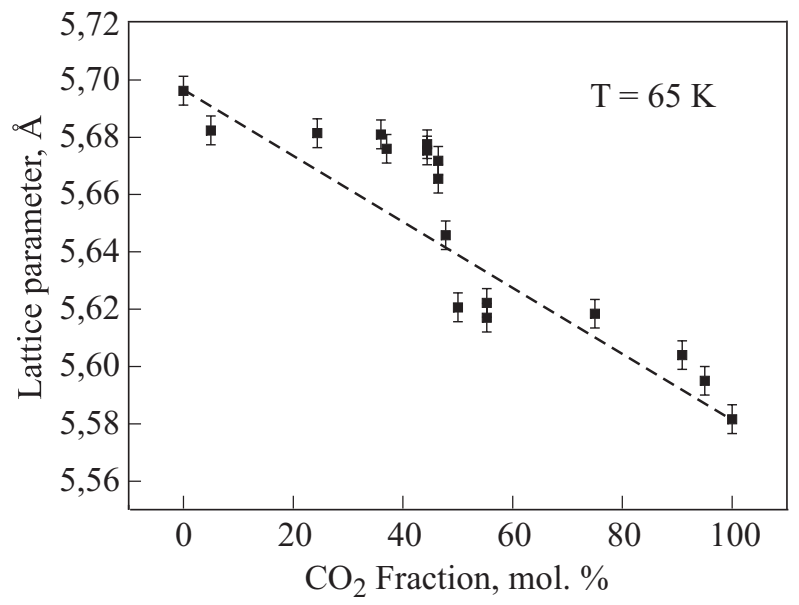

Fig. 2. Concentration dependence of the lattice parameter of solid $\mathrm{CO}_{2}-\mathrm{N}_{2} \mathrm{O}$ mixtures deposited on a substrate at $65 \mathrm{~K}$. Black squares are experimental data. Dashed line - Vegard's curve. result obtained does not allow suggesting the decomposition of the solutions.

The character of electron-diffraction patterns obtained at $5 \mathrm{~K}$ for unannealed low-temperature condensates was found to depend on their composition. For contents around the equimolar composition the diffraction patterns consisted of one halo pointing towards a very high-dispersive or amorphous state of the deposits during the growth process. Reflections were first formed at the positions corresponding to the peaks (111) and (200) for the fcc lattice. The quenching of such samples directly resulted in the formation of the single-phase condensates. Subsequent temperature increase was accompanied by a transition of the system to a higher equilibrium state corresponding $\mathrm{Pa} 3$ structure. Save for the range of equimolar concentration the low temperature quenching of nitrous oxide-rich and carbon dioxide-rich mixtures has led to the formation of the ordered phase. However, a halfwidth of the peaks was slightly larger than for the case of high-temperature deposition. During annealing, a slight increase in grain size was observed even at $40 \mathrm{~K}$. One should be noted the widths of the diffraction maxima for unannealed low temperature deposits of $\mathrm{CO}_{2}-\mathrm{N}_{2} \mathrm{O}$ are narrow enough and they are not characteristics for the binary cryocrystal solutions. From experimental data obtained at $5 \mathrm{~K}$ the lattice parameter of the samples was measured as a function of the impurity mole fraction. The curve of the concentration-dependent lattice parameter is shown in Fig. 3. The lattice parameters of the solid solutions calculated in accordance with the Vegard's rule are shown, too. As a whole the picture has the same character as the high temperature $(T=65 \mathrm{~K})$ dependence. For both low fraction ranges (10 mol.\% impurity and less) the experimental curves are close to the calculated one, while outside these intervals the dependence has a more compli-

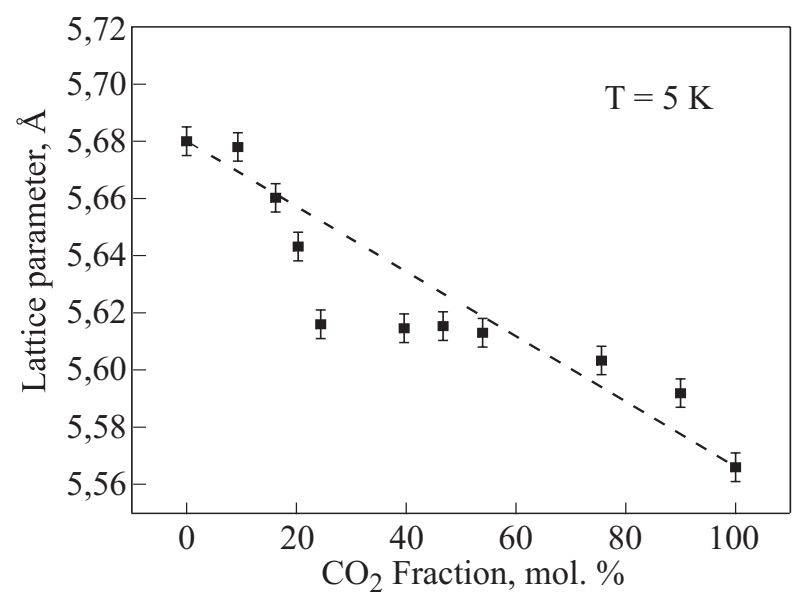

Fig. 3. Concentration dependence of the lattice parameter of solid $\mathrm{CO}_{2}-\mathrm{N}_{2} \mathrm{O}$ mixtures deposited on a substrate at $5 \mathrm{~K}$. Black squares are experimental data. Dashed line - Vegard's curve. 
cated character. The striking variation of the lattice parameter in the narrow concentration interval also takes a place. However this step shifts off and is in the vicinity of 25 mol.\% $\mathrm{CO}_{2}$.

To explain the observable effect, let us assume that $\mathrm{N}_{2} \mathrm{O}$ molecules are shifted off the fcc lattice site along the body diagonal of the cube. The possibility of the displacement of the molecule is related with its asymmetry and owing to this fact there is an uncertainty of its arrangement. There are different models of an arrangement of the non-symmetric linear molecules in the Pa 3 lattice: the fcc site may coincide with the middle of the molecular bond, with the center of the molecular mass and another variants of coincide are possible. According to estimates [14] the displacement from the centers of symmetry of the space group $\mathrm{Pa} 3$ in the $\alpha-\mathrm{CO}$ lattice would produce such small changes in diffraction patterns that their measurements would not show a significant distinction. The probability of the displacement of the $\mathrm{N}_{2} \mathrm{O}$ molecules in the lattice and strong anisotropic interaction of both components facilitate an unlimited solubility in $\mathrm{N}_{2} \mathrm{O}-\mathrm{CO}_{2}$ system.

\section{Conclusions}

The crystallographic structure and the range of existence of sold solutions of the $\mathrm{CO}_{2}-\mathrm{N}_{2} \mathrm{O}$ alloys were determined as a result of THEED data.

It was established that $\mathrm{CO}_{2}$ with $\mathrm{N}_{2} \mathrm{O}$ forms the homogeneous solid solutions in temperature range $5-65 \mathrm{~K}$ at any concentrations and the molecules mutually replace one another in the lattice sites. The structure of solid solutions belongs to the ordered state $P a 3$ symmetry. Like the pure components, the solid solutions do not display the transition from orientationally ordered to disorded phase. In the case of low temperature deposition $(5 \mathrm{~K})$ for the equimolar composition the formation of highly dispersed deposits was observed during the growth process.

The concentration dependencies of the lattice parameter at $T=5 \mathrm{~K}$ and $T=65 \mathrm{~K}$ have the same character and differ from Vegard's approximation. The curves have a step in the values of lattice parameter which is observed at $\mathrm{T}=65 \mathrm{~K}$ for the equimolar composition and shifts to $25 \mathrm{~mol} \% . \mathrm{CO}_{2}$ fraction at helium temperature. A presence of the step means that the range between $\mathrm{N}_{2} \mathrm{O}$-based and $\mathrm{CO}_{2}$-based solutions is a critically narrow. A possible explanation of this phenomenon is proposed. The reconstruction of the solution lattice occurs in conditions where the non-equivalency of the ends of $\mathrm{N}_{2} \mathrm{O}$ molecules and strong anisotropic interaction in the crystals facilitate the transition from $\mathrm{N}_{2} \mathrm{O}$-based to $\mathrm{CO}_{2}$-based solution. In cryocrystals formed from linear 3-atom molecules, the direct anisotropic intermolecular interaction plays a decisive role in the formation of the solid state.

The authors wish to express thanks to M.A. Strzhemechny for stimulating discussions and N.N. Galtsov for help in the work.

1. V.G. Manzhelii and Yu.A. Freiman (eds.), Physics of Cryocrystals, AIP, New York (1996).

2. V.V. Sumarokov, P. Stachowiak, and A. Jezowski, Fiz. Nizk. Temp. 33, 778 (2007) [Low Temp. Phys. 33, 597 (2007)].

3. M.W. Melhuish and R.L. Scott, J. Phys. Chem. 68, 2301 (1964).

4. W.C. Hamilton and M.Petrie, J. Phys. Chem. 65, 1453 (1961).

5. S.I. Kovalenko, E.I. Indan, A.A. Khudoteplaya, and I.N. Krupskii, Phys. Status Solidi A20, 629 (1973).

6. A.M. Tolkachev, V.G. Manzhelii, V.P. Azarenkov, A. Jezowski, and E.A. Kosobutskaya, Fiz. Nizk. Temp. 6, 1533 (1980)[Sov. J. Low Temp. Phys. 6, 1224 (1980)].

7. K.R. Nary, P.L. Kuhns, and M.S. Conradi, Phys. Rew. B26, 3370 (1982).

8. M. Vetter, A. Brodyanski, and H.J. Jodl, Fiz. Nizk. Temp. 33, 1383 (2007) [Low Temp. Phys. 33, 1052 (2007)].

9. M. Vetter, A.P. Brodyanski, S.A. Medvedev, and H.J. Jodl, Phys. Rev. B75, 014305 (2007).

10. M.A. Strzhemechny, A.A. Solodovnik, and S.I. Kovalenko, Fiz. Nizk. Temp. 24, 889 (1998) [Low Temp. Phys. 24, 669 (1998)].

11. A.A. Solodovnik and V.V. Danchuk, Fiz. Nizk. Temp. 29, 1041 (2003) [Low Temp. Phys. 29, 788 (2003)].

12. L. Vegard, Z. Phys. 71, 465 (1932).

13. V.V. Danchuk, A.A. Solodovnik, and M.A. Strzhemechny, Fiz. Nizk. Temp. 33, 783 (2007) [Low Temp. Phys. 33, 600 (2007)].

14. W.N. Lipscomb, J. Chem. Phys. 60, 5138 (1974). 